Complex spherical waves and inverse problems in unbounded domains

This article has been downloaded from IOPscience. Please scroll down to see the full text article.

2006 Inverse Problems 222299

(http://iopscience.iop.org/0266-5611/22/6/023)

The Table of Contents and more related content is available

Download details:

IP Address: 140.112.113.225

The article was downloaded on 23/06/2009 at 13:29

Please note that terms and conditions apply. 


\title{
Complex spherical waves and inverse problems in unbounded domains
}

\author{
Mikko Salo $^{1}$ and Jenn-Nan Wang ${ }^{2}$ \\ ${ }^{1}$ Department of Mathematics and Statistics/RNI, University of Helsinki, PO Box 68, \\ 00014 University of Helsinki, Finland \\ ${ }^{2}$ Department of Mathematics, Taida Institute for Mathematical Sciences and NCTS (Taipei), \\ National Taiwan University, Taipei 106, Taiwan \\ E-mail: mikko.salo@helsinki.fi and jnwang@math.ntu.edu.tw
}

Received 13 July 2006

Published 27 October 2006

Online at stacks.iop.org/IP/22/2299

\begin{abstract}
This work is motivated by the inverse conductivity problem of identifying an embedded object in an infinite slab. The novelty of our approach is that we use complex spherical waves rather than classical Calderón-type functions. For Calderón-type functions, they grow exponentially on one side of a hyperplane and decay exponentially on the other side. Without extra modifications, they are inadequate for treating inverse problems in unbounded domains such as the infinite slab. The obvious reason for this is that Calderón-type functions are not integrable on hyperplanes. So they cannot be used as measurements on infinite boundaries. For complex spherical waves used here, they blow up faster than any given positive polynomial order on the inner side of the unit sphere and decay to zero faster than any given negative polynomial order on the outer side of the unit sphere. We shall construct these special solutions for the conductivity equation in the unbounded domain by a Carleman estimate. Using complex spherical waves, we can treat the inverse problem of determining the object in the infinite slab like the problem in the bounded domain. Most importantly, we can easily localize the boundary measurement, which is of great value in practice. On the other hand, since the probing fronts are spheres, it is possible to detect some concave parts of the object.
\end{abstract}

\section{Introduction}

Special solutions for elliptic equations or systems have played an important role in inverse problems since the pioneering work of Calderón [1]. In 1987, Sylvester and Uhlmann [13] introduced complex geometrical optics solutions to solve the inverse boundary value problem 
for the conductivity equation. Other types of special solutions, called oscillating-decaying solutions, were constructed for general elliptic systems in [10] and [12]. These oscillatingdecaying solutions have been used in solving inverse problems, in particular in detecting inclusions and cavities [10].

In developing the theory for inverse boundary value problems with partial measurements, approximate complex geometrical optics solutions concentrated near hyperplanes and near hemispheres for the Schrödinger equation were given in [3] and [6], respectively. In [6], the construction was based on hyperbolic geometry and was applied in [4] to construct complex geometrical optics solutions for the Schrödinger equation where the real part of the phase function is a radial function, i.e. its level surfaces are spheres. These solutions are called complex spherical waves in [4]. An important feature of these solutions is that they decay exponentially on one side of the sphere and grow exponentially on the other side. The hyperbolic geometry approach does not seem to work for the Laplacian with firstorder perturbations, such as the Schrödinger equation with magnetic potential and isotropic elasticity. Recently, using Carleman estimates, complex spherical waves were constructed for the Schrödinger equation in [8], for the Schrödinger equation with magnetic potential in [2] and for the isotropic elasticity in [14]. The Carleman estimate is a more flexible tool in treating lower order perturbations.

With these complex spherical waves at hand, one can study the inverse problem of reconstructing unknown inclusions or cavities embedded in a body with a known background medium. For the conductivity equation, this problem was documented in [4]. There are several results, both theoretical and numerical, concerning the object identification problem by boundary measurements for the conductivity equation. We will not try to give a full account of these developments here. For detailed references, we refer to [4]. Here we want to point out that there are two striking advantages in using complex spherical waves for object identification problems. On one hand, one can avoid using a unique continuation procedure, more precisely, the Runge approximation, in the reconstruction. On the other hand, by the decaying property of complex spherical waves across the spherical front, the measurements can be easily localized.

The construction of complex spherical waves and the inverse problems studied in the literature cited above were restricted to bounded domains. However, there are many interesting inverse problems whose background domains are unbounded, for instance, identifying an object embedded in an infinite slab. In fact, our work here is motivated by this inverse problem. Ikehata in [5] studied the inverse conductivity problem in an infinite slab where the location of an inclusion is reconstructed by infinitely many boundary measurements. In [5], he used Calderón-type harmonic functions, i.e. $\mathrm{e}^{x \cdot\left(\omega+\mathrm{i} \omega^{\perp}\right)}$ with $\omega \in \mathbb{S}^{n-1}$. These functions are not integrable on hyperplanes. Therefore, they cannot be the Dirichlet data of solutions with finite energy. To avoid this problem, he introduced Yarmukhamedov's Green function to construct a sequence of harmonic functions with finite energy that approximate the Calderóntype function on a bounded part of the slab and are arbitrarily small on an unbounded part of the slab.

In this paper, we use complex spherical waves rather than Calderón-type functions for the inverse problem in the slab. The most obvious advantage is that we do not need Yarmukhamedov's Green function to 'localize' complex spherical waves since we can make these solutions decay faster than any given polynomial order on infinite hyperplanes. Therefore, we can treat the problem in the infinite slab like that in the bounded domain. Furthermore, we can handle the inhomogeneous background medium without requiring the Runge approximation property. Also, since our probing fronts are spheres, we are able to determine some concave parts of the embedded object. 
This paper is organized as follows. In section 2, we discuss a Carleman estimate and its consequence needed in our proof. In section 3, we construct complex spherical waves in the unbounded domain by means of the Carleman estimate. The investigation of the inverse problem in the infinite slab will be discussed in section 4 .

\section{Carleman estimate and related consequence}

As in [2, 8] and [14], we construct complex spherical waves in the unbounded domain via a Carleman estimate. Let $p \in \mathbb{R}^{n}, n \geqslant 2$, be a fixed point. Let $B_{\varepsilon}(p)$ be the ball of radius $\varepsilon>0$ centred at $p$. Without loss of generality, we take $p=0$. We denote $U={\overline{B_{\varepsilon}(0)}}^{c}$. From [2] and [8], we see that $\varphi(x)=\log |x|$ is a limiting Carleman weight for the semiclassical Laplacian $-h^{2} \Delta$ in $U$. Namely, if $a(x, \xi)=|\xi|^{2}-\left|\varphi_{x}^{\prime}\right|^{2}$ and $b(x, \xi)=2 \varphi_{x}^{\prime} \cdot \xi$, then

$$
\{a, b\}(x, \xi)=0 \quad \text { when } \quad a(x, \xi)=b(x, \xi)=0 .
$$

Here, $\{a, b\}=a_{\xi}^{\prime} \cdot b_{x}^{\prime}-a_{x}^{\prime} \cdot b_{\xi}^{\prime}$ is the Poisson bracket of $a, b$. With such $\varphi$, we have

$$
\mathrm{e}^{-\varphi / h}=|x|^{-1 / h} \text {. }
$$

So, $\mathrm{e}^{-\varphi / h}$ becomes a polynomial weight. Carleman estimates with polynomial weights are well known in proving the strong unique continuation property for Schrödinger operators with singular potentials, see, for example, [7]. Since we will work in $L^{2}$-based spaces, it is enough to use a simpler $L^{2}$ Carleman estimate given, for instance, in [11].

Lemma 2.1 [11, lemma 2.1]. There exists $C>0$ such that for any $v \in C_{c}^{\infty}\left(\mathbb{R}^{n} \backslash\{0\}\right)$ and for any $\frac{1}{h} \in\left\{k+\frac{1}{2}: k \in \mathbb{N}\right\}$ with $h \ll 1$, we have

$$
h^{2} \int|x|^{-2 / h}|v|^{2}|x|^{-n} \mathrm{~d} x \leqslant C \int|x|^{-2 / h+4}\left|h^{2} \Delta v\right|^{2}|x|^{-n} \mathrm{~d} x .
$$

Here, the constant $C$ is independent of $v$ and $h$.

Now we replace $1 / h$ by $1 / h-n-\delta$ such that

$$
h \in S:=\left\{\left(k+n+\delta+\frac{1}{2}\right)^{-1}: k \in \mathbb{N}\right\},
$$

where $\delta>0$. Then (2.1) implies that for $v \in C_{c}^{\infty}(U)$ and sufficiently small $h \in S$, we have

$$
h^{2} \int|x|^{-2 / h}|v|^{2}|x|^{n+2 \delta} \mathrm{d} x \leqslant C \int|x|^{-2 / h}\left|h^{2} \Delta v\right|^{2}|x|^{n+4+2 \delta} \mathrm{d} x
$$

with a possibly different constant $C$. Let $q \in L_{c}^{\infty}(U)$ and $P_{h}^{*}=|x|^{-1 / h} h^{2}(\Delta-\bar{q})|x|^{1 / h}$. Then we get from (2.2) that

$$
h\|v\|_{L_{\frac{n}{2}+\delta}^{2}(U)} \leqslant C\left\|P_{h}^{*} v\right\|_{L_{\frac{n}{2}+2+\delta}^{2}(U)}
$$

for all $v \in C_{c}^{\infty}(U)$ and small $h \in S$, where $L_{s}^{2}(U)$ is the weighted $L^{2}$ space with norm $\|f\|_{L_{s}^{2}(U)}=\left\|\langle x\rangle^{s} f\right\|_{L^{2}(U)}$, where $\langle x\rangle=\sqrt{1+|x|^{2}}$. Combining (2.3) and the Hahn-Banach theorem, we prove the following existence theorem.

Proposition 2.2. For any $f \in L_{-\frac{n}{2}-\delta}^{2}(U)$, there exists $w \in L_{-\frac{n}{2}-2-\delta}^{2}(U)$ such that

$$
P_{h} w:=|x|^{1 / h} h^{2}(\Delta-q)\left(|x|^{-1 / h} w\right)=f \quad \text { in } \quad U
$$

and

$$
h\|w\|_{L_{-\frac{n}{2}-2-\delta}^{2}(U)} \leqslant C\|f\|_{L_{-\frac{n}{2}-\delta}^{2}(U)} .
$$


Proof. Let us denote $\mathcal{D}=C_{c}^{\infty}(U)$. For $f \in L_{-\frac{n}{2}-\delta}^{2}(U)$, we define a linear functional $\ell$ on the linear subspace $L=P_{h}^{*} \mathcal{D}$ of $L_{\frac{n}{2}+2+\delta}^{2}(U)$ as follows:

$$
\ell\left(P_{h}^{*} v\right)=\langle v, f\rangle_{L^{2}(U)} \text {. }
$$

Using (2.3), we obtain

$$
\left|\ell\left(P_{h}^{*} v\right)\right| \leqslant\|v\|_{L_{\frac{n}{2}+\delta}^{2}(U)}\|f\|_{L_{-\frac{n}{2}-\delta}^{2}(U)} \leqslant \frac{C}{h}\left\|P_{h}^{*} v\right\|_{L_{\frac{n}{2}+2+\delta}^{2}(U)}\|f\|_{L_{-\frac{n}{2}-\delta}^{2}(U)} .
$$

So by the Hahn-Banach theorem, $\ell$ can be extended to a linear functional on $L_{\frac{n}{2}+2+\delta}^{2}(U)$ with the same norm. Therefore, there exists $w \in L_{-\frac{n}{2}-2-\delta}^{2}(U)$ such that for $v \in C_{c}^{\infty}(\Omega)$

$$
\left\langle P_{h}^{*} v, w\right\rangle_{L^{2}(U)}=\langle v, f\rangle_{L^{2}(U)}
$$

and

$$
h\|w\|_{L_{-\frac{n}{2}-2-\delta}^{2}(U)} \leqslant C\|f\|_{L_{-\frac{n}{2}-\delta}^{2}(U)} .
$$

The proof of the proposition is now complete.

\section{Complex spherical waves in unbounded domains}

In this section we will construct complex spherical waves for the conductivity equation in the unbounded domain $\Omega=\left\{x \in \mathbb{R}^{n}: x_{n}<d\right\}$, where $d<0$. It is clear that $\bar{\Omega} \subset U\left(=\bar{B}_{\varepsilon}(0){ }^{c}\right)$ for some $\varepsilon>0$. Let $0<\gamma(x) \in C^{2}(\bar{\Omega})$ and $\gamma=1$ in $B_{R}(0)^{c} \cap \Omega$ for some $R>0$. We look for a function $v=|x|^{-1 / h} \tilde{v}$ with $h \in S$ satisfying

$$
L v:=\nabla \cdot(\gamma \nabla v)=0 \quad \text { in } \quad \Omega .
$$

Using the Liouville transform $w=\sqrt{\gamma} v$, we know that if $w$ solves

$$
P w:=-\Delta w+q w=0
$$

with $q=\Delta \sqrt{\gamma} / \sqrt{\gamma}$, then $v$ solves (3.1). Here, we note that $q \in L_{c}^{\infty}(\Omega)$. We want to point out that by checking the proofs in [4], complex spherical waves constructed there can be extended to the unbounded domain $\Omega$. In this work, we take a different route using the Carleman estimate which can be adapted to other equations more easily.

As in [2] and [8], we want to construct solutions to $P w=0$ in $\Omega$ which have the form

$$
w=\mathrm{e}^{-\rho(x) / h}(a+r),
$$

where $\rho=\varphi+\mathrm{i} \psi$ and $\varphi, \psi$ are given by

$$
\varphi(x)=\log |x|, \quad \psi(x)=\operatorname{dist}_{\mathbb{S}^{n-1}}\left(\frac{x}{|x|}, e_{1}\right) .
$$

Our first goal is to see what $a$ will look like. To this end, we change coordinates and write $x=\left(x_{1}, x^{\prime}\right)$ where $x^{\prime}=r \theta$ with $r>0$ and $\theta \in \mathbb{S}^{n-2}$. We take $z=x_{1}+$ ir to be a complex variable, and let $\Psi: x \mapsto(z, \theta)$ be the corresponding change of coordinates in $\Omega$. If $f$ is a function in $\Omega$, we write $\tilde{f}=f \circ \Psi^{-1}$. Thus, we can see that

$$
\tilde{\rho}=\log z, \quad(\nabla \rho) \tilde{)}=\frac{1}{z}\left(e_{1}+\mathrm{i} e_{r}\right) \quad \text { with } \quad e_{r}=(0, \theta)
$$

and

$$
(\Delta \rho)^{\tilde{}}=-\frac{2(n-2)}{z(z-\bar{z})}
$$

Also, $\nabla \rho \cdot \nabla$ becomes $(2 / z) \partial_{\bar{z}}$ in the new coordinates. 
Writing $h^{2} P=(-\mathrm{i} h \nabla)^{2}+h^{2} q$, we have

$$
\begin{aligned}
\mathrm{e}^{\rho / h} h^{2} P \mathrm{e}^{-\rho / h} & =(-\mathrm{i} h \nabla+\mathrm{i} \nabla \rho)^{2}+h^{2} q \\
& =-(\nabla \rho)^{2}+h(2 \nabla \rho \cdot \nabla+\Delta \rho)+h^{2} P \\
& =h(2 \nabla \rho \cdot \nabla+\Delta \rho)+h^{2} P .
\end{aligned}
$$

Here, $\varphi$ and $\psi$ were chosen so that $(\nabla \rho)^{2}=0$. To get $P\left(\mathrm{e}^{-\rho / h}(a+r)\right)=0$, we choose $a$ and $r$ to satisfy in $\Omega$

$$
\left(\nabla \rho \cdot \nabla+\frac{1}{2} \Delta \rho\right) a=0
$$

and

$$
\mathrm{e}^{\rho / h} h^{2} P \mathrm{e}^{-\rho / h} r=-h^{2} P a .
$$

The first equation (3.3) is a transport equation for $a$. Writing $a=\tilde{a} \circ \Psi$, this reduces to

$$
\left(\partial_{\bar{z}}-\frac{n-2}{2(z-\bar{z})}\right) \tilde{a}=0 \quad \text { in } \quad \Psi(\Omega) .
$$

It is easy to see that the general solution is given by

$$
\tilde{a}=(z-\bar{z})^{\frac{2-n}{2}} \tilde{g}
$$

for any $\tilde{g}(z, \theta)$ satisfying $\partial_{\bar{z}} \tilde{g}=0$.

Taking $\tilde{g}=1$ and going back to the $x$ coordinates, we obtain

$$
a(x)=\left(2 \mathrm{i}\left|x^{\prime}\right|\right)^{\frac{2-n}{2}}, \quad x \in \Omega .
$$

In (3.4), the right-hand side becomes

$$
-h^{2} P a=(2 \mathrm{i})^{\frac{2-n}{2}} h^{2}\left(\frac{1}{4}(n-2)(4-n)\left|x^{\prime}\right|^{\frac{-2-n}{2}}-q\left|x^{\prime}\right|^{\frac{2-n}{2}}\right) .
$$

This decays in $x^{\prime}$ but is constant in $x_{1}$, so $P a \in L_{s}^{2}(\Omega)$ for $s<-n / 2$. We now rewrite (3.4) as

$$
-|x|^{1 / h} h^{2} P\left(|x|^{-1 / h} \mathrm{e}^{-\mathrm{i} \psi / h} r\right)=\mathrm{e}^{-\mathrm{i} \psi / h} h^{2} P a .
$$

Since $\mathrm{e}^{-\mathrm{i} \psi / h} h^{2} P a \in L_{s}^{2}(\Omega)$ with $s=-n / 2-\delta$ for some $\delta>0$, we deduce from proposition 2.2 that there exists $r$ solving (3.4) and satisfying

$$
\|r\|_{L_{-\frac{n}{2}-2-\delta}^{2}(\Omega)} \leqslant C h .
$$

Therefore, we have constructed the special solution $w$ of $-\Delta w+q w=0$ in $\Omega$ having the form

$$
w=\mathrm{e}^{-(\varphi+\mathrm{i} \psi) / h}(a+r)=|x|^{-1 / h} \mathrm{e}^{-\mathrm{i} \psi / h}(a+r)
$$

with $a$ given by (3.5) and $r$ satisfying (3.6). In particular, we can see that $w \in L^{2}(\Omega)$ for all sufficiently small $h$. Furthermore, since $\Delta w=q w \in L^{2}(\Omega)$, we can use a cut-off technique and the elliptic estimate to show that

$$
w \in H^{2}(\tilde{\Omega}),
$$

where $\tilde{\Omega}=\left\{x \in \mathbb{R}^{n}: x_{n}<\tilde{d}\right\}$ for any $\tilde{d}<d$. In other words, the trace of $w$ on any hyperplane $H_{b}:=\left\{x_{n}=b\right\}$ with $b<\tilde{d}$ is well defined and

$$
\left.\partial_{x_{n}} w\right|_{H_{b}} \in H^{1 / 2} \text {. }
$$

This property is an important difference between complex spherical waves and Calderón-type solutions. 
In order to apply complex spherical waves to the inverse problem, we need to estimate the $H^{1}$ norm of $r$ on any fixed open subset of $\Omega$. Let $\Omega_{1}$ and $\Omega_{2}$ be two open bounded subsets of $\Omega$ such that $\overline{\Omega_{1}} \subset \Omega_{2}$ and $\overline{\Omega_{2}} \subset \Omega$. From (3.2), we see that

$$
\mathrm{e}^{\rho / h} h^{2} P \mathrm{e}^{-\rho / h}=-h^{2} \Delta+h(2 \nabla \rho \cdot \nabla+\Delta \rho)+h^{2} q .
$$

Therefore, $\mathrm{e}^{\rho / h} h^{2} P \mathrm{e}^{-\rho / h}$ is elliptic as a semiclassical operator. Furthermore, we have

$$
\left\|\mathrm{e}^{\rho / h} h^{2} P \mathrm{e}^{-\rho / h} r\right\|_{L^{2}\left(\Omega_{2}\right)}=\left\|h^{2} P a\right\|_{L^{2}\left(\Omega_{2}\right)} \leqslant C h^{2}
$$

and

$$
\|r\|_{L^{2}\left(\Omega_{2}\right)} \leqslant C h \text {. }
$$

Hence, by the semiclassical version of elliptic estimate [9, lemma 2.6], we obtain that

$$
\sum_{|\alpha| \leqslant 2}\left\|h^{\alpha} \partial^{\alpha} r\right\|_{L^{2}\left(\Omega_{1}\right)} \leqslant C h
$$

in particular,

$$
\|r\|_{L^{2}\left(\Omega_{1}\right)}+h\|\nabla r\|_{L^{2}\left(\Omega_{1}\right)} \leqslant C h .
$$

Finally, let $p$ be any point such that $\operatorname{dist}(p, \Omega)>0$, then complex spherical waves for (3.1) in $\Omega$ are given by

$$
v=v(x, h)=\gamma^{-1 / 2}|x-p|^{-1 / h} \mathrm{e}^{-\mathrm{i} \psi / h}(a+r)
$$

for $h \in S$ small, where $\psi=\operatorname{dist}_{\mathbb{S}^{n-1}}\left(\frac{x-p}{|x-p|}, e_{1}\right), a(x)=\left(2 \mathrm{i}\left|x^{\prime}-p^{\prime}\right|\right)^{\frac{2-n}{2}}$ and $r$ satisfies (3.6) and (3.7). Note that for all $x \in \Omega$, we have $x^{\prime}-p^{\prime}=\left(x_{2}-p_{2}, \ldots, x_{n}-p_{n}\right) \neq 0$ since $p_{n}>x_{n}$.

\section{Inverse problem in a slab}

In this section, we shall apply the special solutions we constructed above to the inverse problem in an infinite slab. We study the reconstruction of an embedded cavity here. The same method works for the inclusion case and so does the method in [4] or [14]. We leave this generalization to the interested reader. Let $\Omega=\left\{x \in \mathbb{R}^{n}: d_{1}<x_{n}<d_{2}, d_{1}<d_{2}\right\}$. Assume that $D$ is a bounded domain with a $C^{2}$ boundary in $\Omega$ so that $\bar{D} \subset \Omega$ and $\Omega \backslash \bar{D}$ is connected. Let $u(x)$ be the unique solution of finite energy to

$$
\begin{cases}L_{\gamma} u=0 & \text { in } \quad \Omega \backslash \bar{D}, \\ \gamma \frac{\partial u}{\partial v}=0 & \text { on } \quad \partial D, \\ u=f \in H^{1 / 2} & \text { on } \quad \partial \Omega,\end{cases}
$$

where the conductivity parameter $\gamma(x)$ satisfies the assumptions described in the previous section. The well posedness of (4.1) can be proved by the standard Lax-Milgram theorem with a Poincaré-type inequality in the infinite slab; there exists $C>0$ independent of $u$ such that

$$
\int_{\Omega \backslash \bar{D}}|u|^{2} \mathrm{~d} x \leqslant C \int_{\Omega \backslash \bar{D}}|\nabla u|^{2} \mathrm{~d} x
$$

for all $u \in H^{1}(\Omega \backslash \bar{D})$ with $u=0$ on $x_{n}=d_{1}$ and $d_{2}$. One possible way to prove (4.2) is to divide $\Omega=\bar{Z} \cap(\Omega \backslash \bar{Z})$, where $Z$ is a cylindrical domain such that $\bar{D} \subset Z$. Then, (4.2) is a consequence of

$$
\int_{Z}|v|^{2} \mathrm{~d} x \leqslant C \int_{Z}|\nabla v|^{2} \mathrm{~d} x
$$


for all $v \in H^{1}(Z)$ with $v=0$ on $\partial Z \cap\left\{x_{n}=d_{1}, d_{2}\right\}$ and

$$
\int_{\Omega \backslash \bar{Z}}|w|^{2} \mathrm{~d} x \leqslant C \int_{\Omega \backslash \bar{Z}}|\nabla w|^{2} \mathrm{~d} x
$$

for all $w \in H^{1}(\Omega \backslash \bar{Z})$ with $w=0$ on $\partial(\Omega \backslash \bar{Z}) \cap\left\{x_{n}=d_{1}, d_{2}\right\}$. Now (4.3) can be proved by a contradiction argument and (4.4) can be established by the usual integration technique. For brevity, we left the details to the reader.

The inverse problem here is to identify $D$ from the Dirichlet-to-Neumann map $\Lambda_{D}$ : $H^{1 / 2}(\partial \Omega) \mapsto H^{-1 / 2}(\partial \Omega)$

$$
\Lambda_{D}:\left.f \mapsto \gamma \frac{\partial u}{\partial v}\right|_{\partial \Omega}
$$

In the weak formulation, the Dirichlet-to-Neumann map is defined by

$$
\left\langle\Lambda_{D} f, g\right\rangle=\int_{\Omega \backslash \bar{D}} \gamma \nabla u \cdot \nabla v \mathrm{~d} x,
$$

where $v \in H^{1}(\Omega \backslash \bar{D})$ with $g=\left.v\right|_{\partial \Omega}$. We are interested in the reconstruction problem in this work.

Let $\hat{\Omega}=\left\{x \in \mathbb{R}^{n}: x_{n}<\hat{d}\right\}$ with $d_{2}<\hat{d}$ and $t>0$. Pick any point $p$ so that $\operatorname{dist}(p, \hat{\Omega})>$ 0 . We now denote complex spherical waves of $L_{\gamma} v=0$ in $\hat{\Omega}$ by

$$
v_{t}(x, h)=t^{1 / h} \gamma^{-1 / 2}|x-p|^{-1 / h} \mathrm{e}^{-\mathrm{i} \psi / h}(a+r)=\gamma^{-1 / 2}\left(\frac{t}{|x-p|}\right)^{1 / h} \mathrm{e}^{-\mathrm{i} \psi / h}(a+r) \text {. }
$$

Let us define the energy gap functional

$$
E_{t}(h):=\left\langle\left(\Lambda_{0}-\Lambda_{D}\right) \bar{v}_{t}(x, h), v_{t}(x, h)\right\rangle=\int_{\partial \Omega}\left(\Lambda_{0}-\Lambda_{D}\right) \bar{v}_{t}(x, h) \cdot v_{t}(x, h) \mathrm{d} s,
$$

where $\Lambda_{0}$ is the Dirichlet-to-Neumann map for $L_{\gamma}$ with $D=\emptyset$. Note that $E_{t}(h)$ is well defined for any $t>0$ even when $\partial \Omega$ is unbounded. To understand how we reconstruct $D$, we first observe that $E_{t}(h)$ can be estimated by

$$
\frac{1}{C} \int_{D}\left|\nabla v_{t}(x, h)\right|^{2} \mathrm{~d} x \leqslant E_{t}(h) \leqslant C \int_{D}\left(\left|\nabla v_{t}(x, h)\right|^{2}+\left|v_{t}(x, h)\right|^{2}\right) \mathrm{d} x
$$

for some constant $C>0$.

Proof of (4.5). Let $u$ be the solution of (4.1) with $f=v_{t}$. We observe that

$$
\int_{\Omega \backslash \bar{D}} \gamma \nabla u \cdot \nabla\left(\bar{u}-\bar{v}_{t}\right) \mathrm{d} x=0 .
$$

Using the definition of the Dirichlet-to-Neumann map and (4.6), we can compute

$$
\begin{aligned}
\left\langle\left(\Lambda_{0}-\Lambda_{D}\right) \bar{v}_{t}, v_{t}\right\rangle= & \int_{\partial \Omega} \gamma \frac{\partial \bar{v}_{t}}{\partial v} v_{t} \mathrm{~d} s-\int_{\partial \Omega} \gamma \frac{\partial \bar{u}}{\partial \nu} v_{t} \mathrm{~d} s=\int_{\Omega} \gamma \nabla \bar{v}_{t} \cdot \nabla v_{t} \mathrm{~d} x \\
& -\int_{\Omega \backslash \bar{D}} \gamma \nabla \bar{u} \cdot \nabla v_{t} \mathrm{~d} x=\int_{\Omega \backslash \bar{D}} \gamma \nabla \bar{v}_{t} \cdot \nabla v_{t} \mathrm{~d} x-\int_{\Omega \backslash \bar{D}} \gamma \nabla \bar{u} \cdot \nabla v_{t} \mathrm{~d} x \\
& +\int_{\Omega \backslash \bar{D}} \gamma \nabla u \cdot \nabla\left(\bar{u}-\bar{v}_{t}\right) \mathrm{d} x+\int_{D} \gamma\left|\nabla v_{t}\right|^{2} \mathrm{~d} x \\
= & \int_{D} \gamma\left|\nabla v_{t}\right|^{2} \mathrm{~d} x+\int_{\Omega \backslash \bar{D}} \gamma \nabla\left(u-v_{t}\right) \cdot \nabla\left(\bar{u}-\bar{v}_{t}\right) \mathrm{d} x
\end{aligned}
$$

Formula (4.7) immediately implies the first inequality of (4.5). 
To obtain the second inequality of (4.5), we need to estimate the last term in (4.7). Denote $w=u-v_{t}$. By construction, we have that

$$
\begin{cases}L_{\gamma} w=0 & \text { in } \quad \Omega \backslash \bar{D}, \\ \gamma \frac{\partial w}{\partial v}=-\gamma \frac{\partial v_{t}}{\partial v} & \text { on } \quad \partial D, \\ w=0 & \text { on } \quad \partial \Omega .\end{cases}
$$

It follows from the elliptic regularity theorem that

$$
\|w\|_{H^{1}(\Omega \backslash \bar{D})} \leqslant C\left\|\gamma \frac{\partial v_{t}}{\partial v}\right\|_{H^{-1 / 2}(\partial D)} .
$$

On the other hand, we know that $L_{\gamma} v_{t}=0$ in $D$ and therefore,

$$
\left\|\gamma \frac{\partial v_{t}}{\partial v}\right\|_{H^{-1 / 2}(\partial D)} \leqslant C\left\|v_{t}\right\|_{H^{1}(D)} .
$$

Putting together (4.8) and (4.9) leads to

$$
\|w\|_{H^{1}(\Omega \backslash \bar{D})} \leqslant C\left\|v_{t}\right\|_{H^{1}(D)}
$$

and the second estimate in (4.5). t's.

Now we will use (4.5) to study the behaviours of $E_{t}(h)$ as $h \rightarrow 0$ and $h \in S$ for different

Theorem 4.1. Let $\operatorname{dist}(D, p)=: d_{0}>0$. For $t>0$ and sufficiently small $h \in S$, we have that

(i) if $d_{0}>t$, then $E_{t}(h) \leqslant C \alpha^{1 / h}$ for some $\alpha<1$;

(ii) if $d_{0}<t$, then $E_{t}(h) \geqslant C \beta^{1 / h}$ for some $\beta>1$ and

(iii) if $\bar{D} \cap \overline{B_{t}(p)}=\{y\}$, then $C^{-1} h^{n-2} \leqslant E_{t}(h) \leqslant C h^{-1}$ for some $C>0$.

\section{Proof.}

(i) If $\operatorname{dist}(D, p)=d_{0}>t$, then

$$
\frac{t}{|x-p|}<1 \quad \forall x \in \bar{D} .
$$

Combining the second inequality of (4.5) and the behaviour of $v_{t}(x, h)$, we immediately prove this statement.

(ii) We first pick a small ball $B_{\delta} \subset \subset B_{t}(p) \cap D$. Using (3.7), the leading term of $\nabla v_{t}(x, h)$ is

$$
-\frac{1}{h}\left(\frac{t}{|x-p|}\right)^{1 / h} \mathrm{e}^{-\mathrm{i} \psi / h} \gamma^{-1 / 2} \nabla(\log |x-p|+\mathrm{i} \psi) a .
$$

We note that $\gamma^{-1 / 2} \nabla(\log |x-p|+\mathrm{i} \psi) a \neq 0$ for all $x \in B_{\delta}$. So this statement follows from the first inequality of (4.5) and the fact

$$
\frac{t}{|x-p|}>1 \quad \forall x \in B_{\delta} \text {. }
$$


(iii) Pick a small cone with vertex at $y$, say $\Gamma$, so that there exists $\epsilon>0$ satisfying

$$
\Gamma \cap\{0<|x-y|<\epsilon\} \subset D .
$$

It is not restrictive to take $y=0$ and $p=(0, \ldots, 0, t)$ for $t>0$. Now we observe that if $z \in \Gamma$ and $|z-y|=|z|=s<\epsilon$, then

$$
|z-p| \leqslant s+t
$$

that is,

$$
\frac{t}{|z-p|} \geqslant \frac{t}{s+t}
$$

Hence, from the first inequality of (4.5) and (4.10), for $h \ll 1$ we have that

$$
\begin{aligned}
E_{t}(h) & \geqslant \frac{C}{h^{2}} \int_{0}^{\epsilon}\left(\frac{t}{s+t}\right)^{2 / h} s^{n-1} \mathrm{~d} s \\
& \geqslant \frac{C}{h^{2}} \sum_{k=1}^{n} \frac{h}{2-k h} \\
& \geqslant C h^{n-2} .
\end{aligned}
$$

On the other hand, we can choose a cone $\widetilde{\Gamma}$ with vertex at $p$ such that $\bar{D} \subset \widetilde{\Gamma} \cap\{t \leqslant$ $|x-p|<t+\eta\}$ for $\eta>0$. Thus, by the second inequality of (4.5) and (4.10), we can estimate

$$
E_{t}(h) \leqslant C(t+\eta)^{n-1} \frac{1}{h^{2}} \int_{t}^{t+\eta}\left(\frac{t}{s}\right)^{2 / h} \mathrm{~d} s \quad \leqslant C h^{-1} .
$$

The distinct behaviour of $E_{t}(h)$ immediately allows us to detect the boundary of the cavity. However, theorem 4.1 is unpractical since we need to take the measurements on the whole unbounded boundary. Fortunately, taking advantage of the decaying property of complex spherical waves, we are able to localize the measurement near the part where complex spherical waves are not decaying. To be precise, let $\phi_{\delta, t}(x) \in C_{0}^{\infty}\left(\mathbb{R}^{n}\right)$ satisfy

$$
\phi_{\delta, t}(x)=\left\{\begin{array}{lll}
1 & \text { on } & B_{t+\delta / 2}(p) \\
0 & \text { on } & \mathbb{R}^{n} \backslash \overline{B_{t+\delta}(p)}
\end{array}\right.
$$

where $\delta>0$ is sufficiently small. Now we are going to use the measurement $f_{\delta, t}(x, h)=$ $\left.\phi_{\delta, t} v_{t}(x, h)\right|_{\partial \Omega}$. Clearly, the measurement $f_{\delta, t}$ is localized on $B_{t+\delta}(p) \cap \partial \Omega$. In fact, if $\delta$ is sufficiently small, $f_{\delta, t}$ is localized on only one part of $\partial \Omega$, depending on whether $p$ lies above or below $\Omega$. Let us define

$$
E_{\delta, t}(h)=\left\langle\left(\Lambda_{0}-\Lambda_{D}\right) \bar{f}_{\delta, t}, f_{\delta, t}\right\rangle
$$

Theorem 4.2. The statements of theorem 4.1 are valid for $E_{\delta, t}(h)$.

Proof. The main idea is to prove that the error caused by the remaining part of the measurement $g_{\delta, t}:=\left.\left(1-\phi_{\delta, t}\right) v_{t}(x, h)\right|_{\partial \Omega}$ is as small as any given polynomial order. Let $w_{\delta, t}(x, h)$ be the unique solution of

$$
L_{\gamma} w=0 \quad \text { in } \quad \Omega
$$

with boundary value $g_{\delta, t}$. We now want to compare $w_{\delta, t}$ with $\left(1-\phi_{\delta, t}\right) v_{t}$. To this end, we first observe that

$$
\begin{cases}L_{\gamma}\left(\left(1-\phi_{\delta, t}\right) v_{t}-w_{\delta, t}\right)=L_{\gamma}\left(\left(1-\phi_{\delta, t}\right) v_{t}\right) & \text { in } \Omega, \\ \left(1-\phi_{\delta, t}\right) v_{t}-w_{\delta, t}=0 & \text { on } \partial \Omega .\end{cases}
$$


Note that

$L_{\gamma}\left(\left(1-\phi_{\delta, t}\right) v_{t}\right)=\left(1-\phi_{\delta, t}\right) L_{\gamma} v_{t}+\left[L_{\gamma},\left(1-\phi_{\delta, t}\right)\right] v_{t}=\left[L_{\gamma},\left(1-\phi_{\delta, t}\right)\right] v_{t}$.

So we see that

$$
\operatorname{supp}\left(L_{\gamma}\left(\left(1-\phi_{\delta, t}\right) v_{t}\right)\right) \subset \bar{\Omega} \cap\{t+\delta / 2 \leqslant|x-p| \leqslant t+\delta\}
$$

and therefore

$$
\left\|L_{\gamma}\left(\left(1-\phi_{\delta, t}\right) v_{t}\right)\right\|_{L^{2}(\Omega)} \leqslant C \alpha_{1}^{1 / h}
$$

for some $0<\alpha_{1}<1$. Consequently, we have that

$$
\left\|\left(1-\phi_{\delta, t}\right) v_{t}-w_{\delta, t}\right\|_{H^{1}(\Omega)} \leqslant C \alpha_{1}^{1 / h},
$$

in particular,

$$
\left\|\left(1-\phi_{\delta, t}\right) v_{t}-w_{\delta, t}\right\|_{H^{1}(D)} \leqslant C \alpha_{1}^{1 / h} .
$$

Using (4.5) for $\left\langle\left(\Lambda_{0}-\Lambda_{D}\right) \bar{g}_{\delta, t}, g_{\delta, t}\right\rangle$ with $v_{t}$ being replaced by $w_{\delta, t}$, we get from (4.11) and decaying property of $v_{t}$ that

$$
\left\langle\left(\Lambda_{0}-\Lambda_{D}\right) \bar{g}_{\delta, t}, g_{\delta, t}\right\rangle \leqslant C \alpha_{2}^{1 / h}
$$

for some $0<\alpha_{2}<1$.

Now we first consider (i) of theorem 4.1 for $E_{\delta, t}(h)$. We shall use a trick given in [4]. In view of the definition of the energy gap functional and the proof of the first inequality of (4.5), we get that

$$
0 \leqslant\left\langle\left(\Lambda_{0}-\Lambda_{D}\right)\left(\zeta \bar{f}_{\delta, t} \pm \zeta^{-1} \bar{g}_{\delta, t}\right), \zeta f_{\delta, t} \pm \zeta^{-1} g_{\delta, t}\right\rangle
$$

for any $\zeta>0$, which leads to

$$
\begin{aligned}
& \left|\left\langle\left(\Lambda_{0}-\Lambda_{D}\right) \bar{f}_{\delta, t}, g_{\delta, t}\right\rangle+\left\langle\left(\Lambda_{0}-\Lambda_{D}\right) \bar{g}_{\delta, t}, f_{\delta, t}\right\rangle\right| \\
& \quad \leqslant \zeta^{2}\left\langle\left(\Lambda_{0}-\Lambda_{D}\right) \bar{f}_{\delta, t}, f_{\delta, t}\right\rangle+\zeta^{-2}\left\langle\left(\Lambda_{0}-\Lambda_{D}\right) \bar{g}_{\delta, t}, g_{\delta, t}\right\rangle .
\end{aligned}
$$

It now follows from $\left.v_{t}(x, h)\right|_{\partial \Omega}=f_{\delta, t}+g_{\delta, t}$ and (4.12) with $\zeta=1 / \sqrt{2}$ that

$$
\begin{aligned}
\frac{1}{2}\left\langle\left(\Lambda_{0}-\Lambda_{D}\right) \bar{f}_{\delta, t}, f_{\delta, t}\right\rangle & \leqslant\left\langle\left(\Lambda_{0}-\Lambda_{D}\right) \bar{g}_{\delta, t}, g_{\delta, t}\right\rangle+\left\langle\left(\Lambda_{0}-\Lambda_{D}\right) \bar{v}_{t}, v_{t}\right\rangle \\
& \leqslant C \alpha_{2}^{1 / h}+\left\langle\left(\Lambda_{0}-\Lambda_{D}\right) \bar{v}_{t}, v_{t}\right\rangle .
\end{aligned}
$$

So from (i) of theorem 4.1, the same statement holds for $E_{\delta, t}(h)$. Likewise, the second inequality of (iii) in theorem 4.1 also holds.

Next, we consider (ii) and the first inequality of (iii) in theorem 4.1 for $E_{\delta, t}$. Choosing $\zeta=1$ in (4.12), we get that

$$
\begin{aligned}
\frac{1}{2}\left\langle\left(\Lambda_{0}-\Lambda_{D}\right) \bar{v}_{t}, v_{t}\right\rangle & \leqslant\left\langle\left(\Lambda_{0}-\Lambda_{D}\right) \bar{g}_{\delta, t}, g_{\delta, t}\right\rangle+\left\langle\left(\Lambda_{0}-\Lambda_{D}\right) \bar{f}_{\delta, t}, f_{\delta, t}\right\rangle \\
& \leqslant C \alpha_{2}^{1 / h}+\left\langle\left(\Lambda_{0}-\Lambda_{D}\right) \bar{f}_{\delta, t}, f_{\delta, t}\right\rangle .
\end{aligned}
$$

Therefore, (ii) of theorem 4.1 and (4.14) implies that the same fact holds for $E_{\delta, t}$. Likewise, the first inequality of (iii) in theorem 4.1 holds for $E_{\delta, t}$. The proof is now complete.

Remark 4.3. With the help of theorem 4.2, when parts of $\partial D$ are near the boundary $\partial \Omega$, we could detect some partial information of $\partial D$ from only a few measurements taken from a very small region on one side of $\partial \Omega$.

To end the presentation, we provide an algorithm of the method.

Step 1

Pick a point $p \notin \bar{\Omega}$ and near $\partial \Omega$. Construct complex spherical waves $v_{t}(x, h)$ for $h \in S$. 
Step 2

Draw two balls $B_{t}(p)$ and $B_{t+\delta}(p)$. Set the Dirichlet data $f_{\delta, t}=\left.\phi_{\delta, t} v_{t}\right|_{\partial \Omega}$. Measure the Neumann data $\Lambda_{D} f_{\delta, t}$ over the region $B_{t+\delta}(p) \cap \partial \Omega$.

Step 3

Calculate $E_{\delta, t}(h)=\left\langle\left(\Lambda_{0}-\Lambda_{D}\right) \bar{f}_{\delta, t}, f_{\delta, t}\right\rangle$. If $E_{\delta, t}(h)$ tends to zero as $h \rightarrow 0$, then the probing front $\{|x-p|=t\}$ does not intersect the inclusion. Increase $t$ and compute $E_{\delta, t}(h)$ again.

Step 4

If $E_{\delta, t}(h)$ increases to $\infty$ as $h \rightarrow 0$, then the front $\{|x-p|=t\}$ intersects the inclusion. Decrease $t$ to make a more accurate estimate of $\partial D$.

Step 5

Choose a different $p$ and repeat the steps $1-4$.

\section{Acknowledgments}

Mikko Salo was partially supported by the Academy of Finland. Jenn-Nan Wang was supported in part by the National Science Council of Taiwan (NSC 94-2115-M-002-003). This work was done when both authors were visiting the University of Washington. We would like to thank Gunther Uhlmann for his encouragements and the Department of Mathematics at the University of Washington for its hospitality.

\section{References}

[1] Calderón A 1980 On an inverse boundary value problem Seminar on Numerical Analysis and its Applications to Continuum Physics, Soc. Brasileira de Matemática (Río de Janeiro) pp 65-73

[2] Dos Santos Ferreira D, Kenig C, Sjöstrand J and Uhlmann G 2006 Determining the magnetic Schrödinger operator from partial Cauchy data Comm. Math. Phys. to appear (Preprint math.AP/0601466)

[3] Greenleaf A and Uhlmann G 2001 Local uniqueness for the Dirichlet-to-Neumann map via the two-plane transform Duke Math. J. 108 599-617

[4] Ide T, Isozaki H, Nakata S, Siltanen S and Uhlmann G 2005 Probing for electrical inclusions with complex spherical waves Commun. Pure Appl. Math. to appear

[5] Ikehata M 2001 Inverse conductivity problem in the infinite slab Inverse Problems 17 437-54

[6] Isozaki H and Uhlmann G 2004 Hyperbolic geometry and the local Dirichlet-to-Neumann map Adv. Math. 188 294-314

[7] Jerison D and Kenig C 1985 Unique continuation and absence of positive eigenvalues for Schrödinger operators Ann. Math. 121 463-94

[8] Kenig C, Sjöstrand J and Uhlmann G 2004 The Calderón problem with partial data Ann. Math. submitted (Preprint math.AP/0405486)

[9] Koch H, Tataru D and Zworski M 2006 Semiclassical $L^{p}$ estimates Ann. H Poincaré to appear (Preprint math-ph/0603080)

[10] Nakamura G, Uhlmann G and Wang J-N 2005 Oscillating-decaying solutions, Runge approximation property for the anisotropic elasticity system and their applications to inverse problems J. Math. Pures Appl. 84 21-54

[11] Regbaoui R 1997 Strong uniqueness for second order differential operators J. Funct. Anal. 141 201-17

[12] Nakamura G, Uhlmann G and Wang J-N Oscillating-decaying solutions for elliptic systems Contemp. Math.

[13] Sylvester J and Uhlmann G 1987 A global uniqueness theorem for an inverse boundary value problem Ann. Math. 125 153-69

[14] Uhlmann G and Wang J-N Complex spherical waves for the elasticity system and probing of inclusions SIAM J. Math. Anal. to appear 